DE92 016373

\title{
JACOBIAN AND STIFFNESS ANALYSIS OF A NOVEL CLASS OF SIX-DOF PARALLEL MINIMANIPULATORS
}

\author{
Farhad Tahmasebi \\ Robotics Branch \\ NASA \\ Goddard Space Flight Center \\ Greenbelt, MD 20771 \\ Associate Member of ASME
}

\author{
Lung-Wen Tsai \\ Mechanical Engineering Dept. \\ and Systems Research Center \\ University of Maryland \\ College Park, MD 20742 \\ Fellow A.SMS
}

\begin{abstract}
The Jacobian and t: ass matrices of two types of novel, sixDOF parallel minimain pulators are derived. A minimanipulator consists of three inextensible limbs, each of which is driven by a two-DOF driver. Bilinear stepper motors are used as drivers in the first type minimanipulator, whereas five-bar linkages are used as drivers in the second type minimanipulator. All of the minimanipulator actuators are hase-mounted. Inextensible limbs (and five-bar linkage drivers in the second type minimanipulator) improve positional resolution and stiffness of the minimanipulators in certain directions. It is shown that, at the central configuration, the stiffness matrix of the first type minimanip. ulator can be diagonalized (decoupled). It is also shown that lie first wpe minimanipulator can be designed to possess direcl or torsional isotropic stiffness properties. Moreover. guidelines for designing the drivers of the second type minimanipulator are established.
\end{abstract}

\section{INTRODUCTION}

Parallel mechanisms have been used for applications in which the requirements for accuracy, rigidity, and load-to-weight ratio are more important than the need for a large workspace.

Stewart (1965) introduced his famous six-degree-of-freedom (six-DOF) platform as a motion simulator. Recently, many researchers have suggested the Stewart platform as a rob" manipulator (e.g., Hunt, 1983; Fichter, 1986). Other six-DOi parallel manipulators have also been introduced and studied in h.e lit. erature (e.g., Kohli $r$ - al., 1985; Hudgens and Tesar, 1988; 'Tsai and Tahmascbi, 1yy,a).

Dualities of serial and parallel manipulators were demonstrated by Waldron and Hunt (1987). For example, inverse kineriatics of a serial manipulator ss much more difficult than its direct kinematics; whereas, for a parallel manipulator, the opposite is true. Closed-form solutions have been obtained for direct kinematics of certain parallel manipulators (e.g., Griffis and Duffy, 1989; Nanua et al., 1990; Innocenti and ParentiCastelli, 1990; Tahmasebi and Tsai, 1991).

Gosselin and Angeles $(1988,1989)$ considered isotropy of the Jacobian matrix for design optimization of planar and spherical three-DOF parallel manipulators. Arai et al. (1990) also used the Jacobian matrix in optimal design of a six-DOF parallel manipulator. Stiffness matrices of parallel manipulators. which are closely related to their Jacobian matrices, have been studied by Kerr (1989) and Gosselin (1990).

$A$ class of three-limbed, six-DOF parallel minimanipulators were introduced by Tsai and Tahmasebi (1991a, 1991b) to obtain high positioning resolution and high stiffness in fine. matupulation operacions. ' In this paper, the Jacobian and stiflness matrices are derived for the minimanipulators. In addition, the Jacobian and stiffness matrices are used in establishing design guidelines for the minimanipulators.

\section{DESCRIPTION OF THE MINIMANIPULATORS}

Let subscript $i$ in this section and the rest of this work represent numbers 1,2 , and 3 in a cyclic manner. A minimanipulator contains three inextensible limbs, $P_{i} R_{i}$, as shown in Figure 1. The lower end of each limb is connected to a two-DOF planar driver and can be moved freely on the base plate. The desired minimanipulator $\mathrm{m}$-tion is obtained by moving the lower ends of its three limbs on its base plate. Two-DOF universal joints connect the limbs to the moving platform. The lower ends of the limbs are connected to the drivers through three more uni. versal joints. Note that one of the axes of the upper universal joint is collinear with the limb, while the other axis of the upper universal joint as well as one of the axes of the lower universal

\footnotetext{
iA patent application has been fited for the minimanipulator:
}

This paper has been accepted for presentation at the 22nd ASME Biennial Mechansms Conference (September 1992). Scotto dale, $A Z$ 


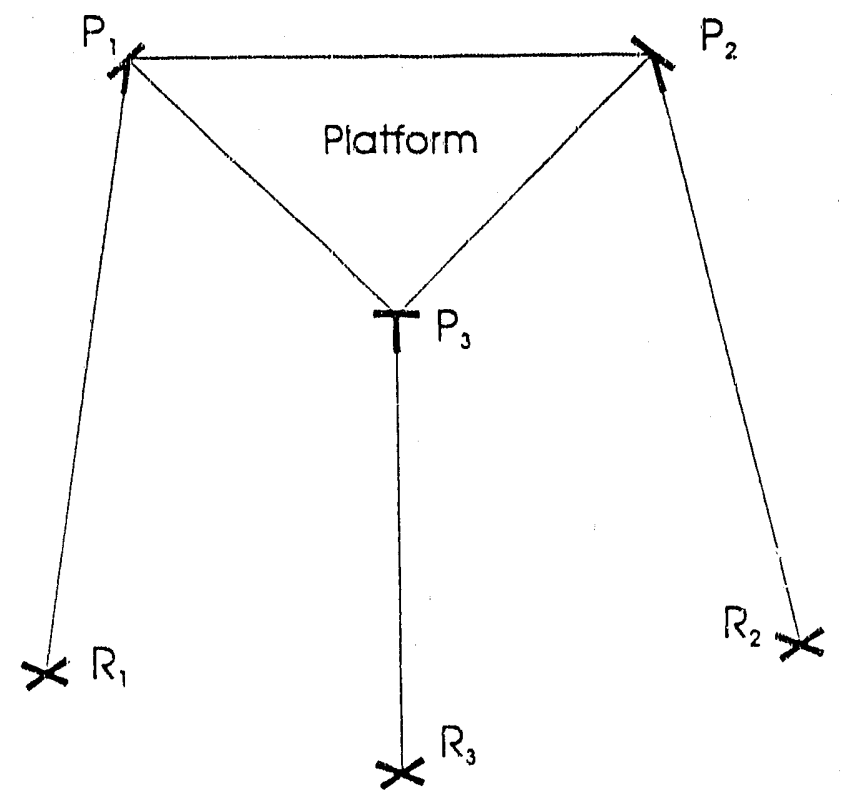

$R_{1}, R_{2}$ and $R_{3}$ are connected to drivers.

Figure 1: Representalion of a minimanipulator

joint are always perpendicular to the limb. This arrangement is kinematirally equivalent to a limb with a spherical joint at its lower end and a revolute joint at its upper end, as shown in Figure 2. In this paper, two types of minimanipulators are studied. The difference between Type 1 minimanipulators and Type 2 minimanipulators is in their two-DOF drivers.

In the Type 1 minimanipulator, bidirectional linear stepper motors (Y'caple, 19S8) are used as drivers. Such stepper motors act as $X-Y^{\prime}$ positioning tables, but their stators are bascmounted.

In the Type 2 minimanipulator, simplified five-bar linkage are ueed as drivers. Figure 3 shows drivers for the Type 2 minimanipulator. Point $C_{i}$ is the output point of a driver. At point $D_{i}$, there is an actuator on each side of the base plate to drive links $D_{i} A_{i}$ and $D_{i} B_{i}$. The simplified five-bar drivers are completely symmetric. That is

$$
\begin{aligned}
& \left|\overline{D_{i} A_{i}}\right|=\left|\overline{D_{i} B_{i}}\right|=a \\
& \left|\overline{A_{i} C_{i}}\right|=\left|\overline{B_{i} C_{i}}\right|=b
\end{aligned}
$$

As a result, coordination between actuator rotations can be easily accomplished. Namely, ingular displacement of an output point $C_{i}$ is obtained by equal actuator rotations, and its radial displacement is obtained by equal and opposite actuator rotations.

Inextensible limbs and simplitiod five-bar linkages (in the Type 2 minimanipulator) are used to improve positional resolu. tion and stiffness of a minimanipulator. Since the minimanipulator actuators are base-mounted; higher payload capacity, smaller actuator sizes, and lower power dissipation can be obtained. In addition, to achicve even load distribution, the minimanipulators are made completely symmetric. Namely, both triangles $D_{1} D_{2} D_{3}$ and $P_{1} P_{2} P_{3}$ are made equilateral and the joint axes at

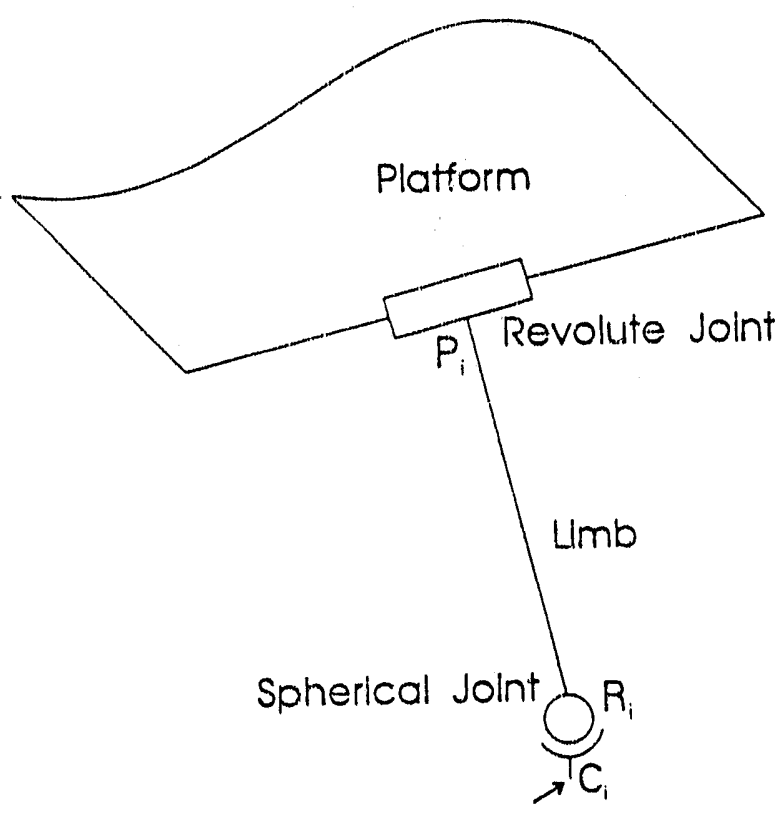

Output Point of a TwO-DOF Driver

Figure 2: linematic equivalent of a limb

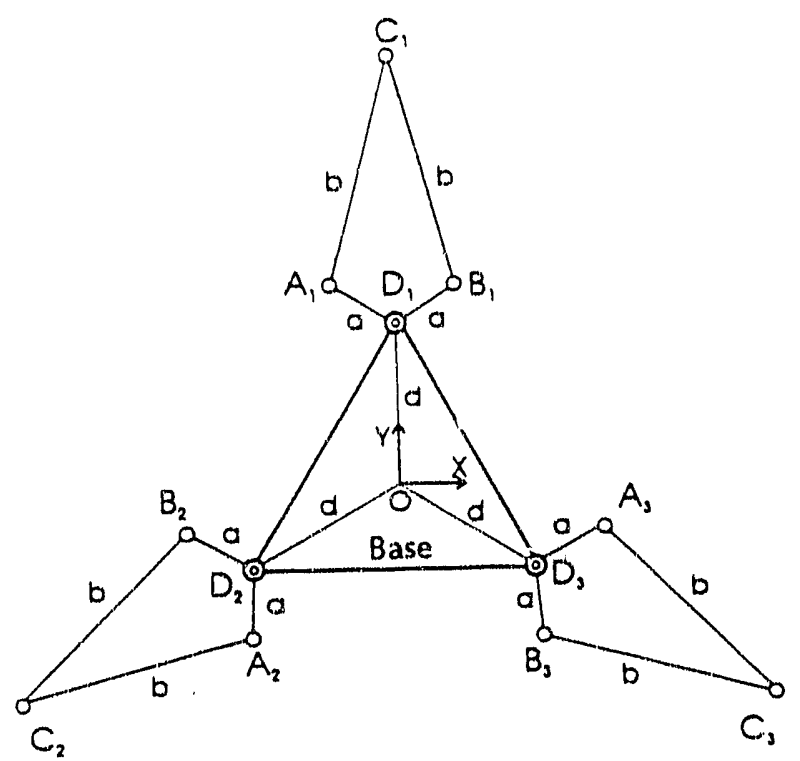

Figure 3: Simplified five-bar linkage drivers 


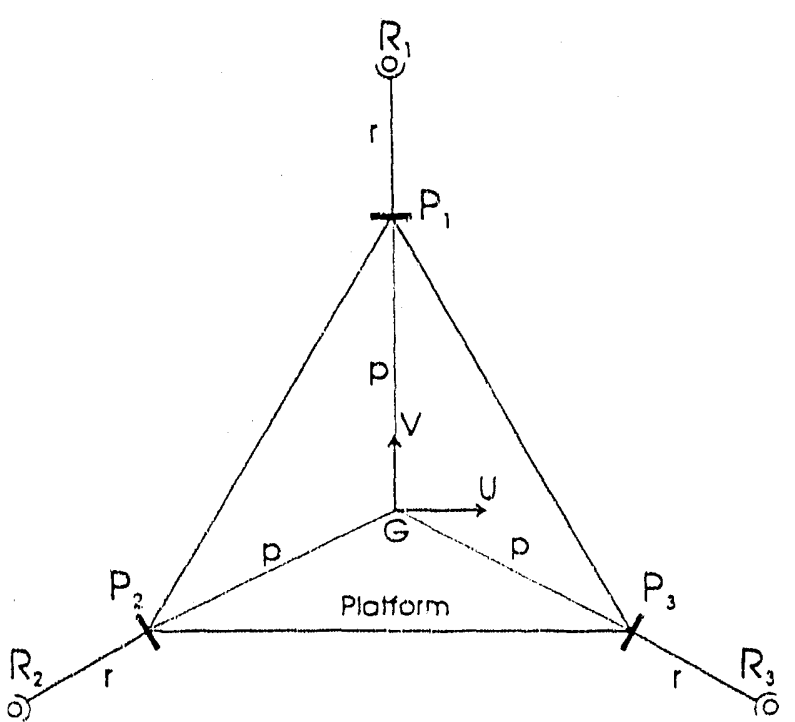

$R_{1}, R_{2}$, and $R_{3}$ are connected to drivers.

Figure 4: Kinematic equivalent of a minimanipulator

points $P_{1}, P_{2}$ and $P_{3}$ are made parallel on lines $P_{2} P_{3}, P_{1} P_{3}$, and $P_{1} P_{2}$, respectively.

In addition to the drivers mentioned above, other twoDOF mechanisms such as regular five-bar linkages, pantographs, or X.Y positioning tables can also be used as drivers for a minimanipulator (Tsai and Tahmasebi, 1991a).

\section{TYPE 1 MINIMANIPULATOR}

In this section, expressions for the Jacobian and stiffness matrices of the Type 1 minimanipulator are derived.

\subsection{Jacobian Analysis}

First, let us define a fixed reference frame (XYZ) and a moving reference frame (UVW). As shown in Figure 3, the fixed (or base) reference frame is attached to the basc. The origin of the fixed reference frame (point $O$ ) is placed at the centroid of triangle $D_{1} D_{2} D_{3}$. The positive $X$-axis is parallel to and points in the direction of vector $\overline{D_{2} D_{3}}$. The positive $Y$-axis points from poins $O$ to point $D_{1}$. The $Z$-axis is defined by the right-handrule. The moving (or platform) reference frame is altached to the platfotm. The origin of the moving reference frame (point $G_{3}$ ) is placed at the centroid of triangle $P_{1} P_{2} P_{3}$ (see Figure 4). The positive U-axis is parallel to and points in the direction of vector $\overline{P_{2} P_{3}}$. The positive $V$-axis points from point $O$ to point $P_{1}$. The $W$-axis is defined by the right-hand-rule.

In this paper, without loss of generality, we let $Z_{R, i}=0$. If $Z_{R_{1 .}}>0$ for a minimanipulator, a simpie transformation should bo: applied to the coordinates of the poirts used in the following derivations.

Referring to Figure 5, we can wrice the following vector equation

$$
\overline{O R_{1}}=\overline{O G}+\overline{G P_{1}}+\overline{P_{1} R_{1}}
$$

Taking the tume derivative of both sides of equation (3) with

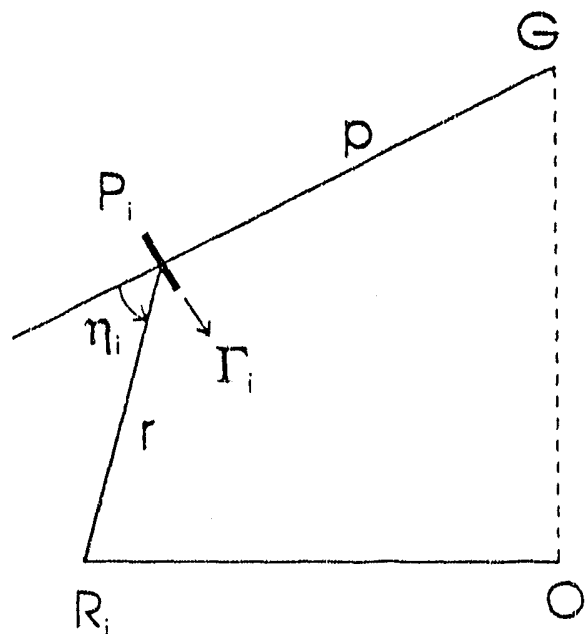

Figure 5: Parameters used in Jacobian analysis

respect to the fixed reference frame yields

$$
{ }^{B} \bar{V}^{R_{i}}={ }^{B} \bar{V}^{G}+{ }^{B} \bar{\omega}^{P} \times \overline{G P_{i}}+{ }^{B} \bar{\omega}^{L_{i}} \times \overline{P_{i} R_{i}}
$$

where $\bar{V}$ and $\bar{w}$ denote linear and angular velocities, respectively. The right superscript for a velocity vector stands for a point or a rigid body, whereas the left superscript refers to a reference frame in which the velocity is expressed. The base, the platform, and limb $\overline{P_{i} R_{i}}$ reference frames (rigid bodies) are denoted by $B$, $P$, and $L_{i}$, respectively. The terms rigid body and reference frame are used interchangeably, because every rigid body can be used as a reference frame and every reference frame can be viewed as a massless rigid body (Kane and Levi, on, 1985). Angular velocity of limb $\overline{P_{i} R_{i}}$ in the fixed reference frame can be found from

$$
{ }^{B} \bar{\omega}^{L_{1}}=P^{P} \bar{\omega}^{L_{1}}+B_{\omega^{P}}^{P}
$$

As shown in Figure 5, let $\eta_{i}$ be the angle from vector $\overline{G P_{i}}$ to vector $\bar{P}_{i} R_{i}$ measured ahout a unit vector $\bar{\Gamma}_{i}$ which is collinear with the axis of the revolute joint at point $P_{1}$ and points in the direction of vector $\overline{P_{i+2} P_{1+1}}$. 2 Then

$$
B_{\bar{\omega}} L_{1}=\dot{\eta}_{i} \bar{\Gamma}_{1}+{ }^{B} \bar{\omega}^{P}
$$

where $\dot{\eta}_{i}$ is the time-derivative of $\eta_{i}$. Substituting the above expression for ${ }^{B} \bar{\omega}^{L_{1}}$ in equation (4), and simplifying, we obtain

$$
{ }^{B} \bar{V}^{R_{1}}={ }^{B} \bar{V}^{G}+{ }^{B} \bar{\omega}^{P} \times \overline{G R_{i}}+\dot{\eta}_{i} \bar{\Gamma}_{i} \times \overline{P_{1} R_{i}}
$$

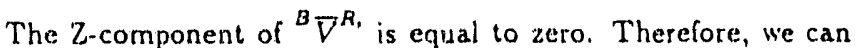
conclude from the above equation that

$$
\dot{n}_{i}=-\frac{\bar{V}^{G} \cdot \bar{n}_{2}+\left({ }^{B} \bar{w}^{P} \times \overline{G R_{1}}\right) \cdot \ddot{n}_{2}}{\left(\bar{\Gamma}_{1} \times \overline{P_{1} R_{i}}\right) \cdot \ddot{n}_{2}}
$$

where $\bar{n}_{x}$ is a unit vector in the Z-direction. Let

$$
\bar{\mu}_{i}=\bar{\Gamma}_{1} \times \overline{P_{1} R_{1}}
$$

\footnotetext{
${ }^{2}$ The subseripts are cyclic, with a cycle length of 3 .
} 
Then, equation (8) reduces to

$$
\dot{\eta}_{i}=-\frac{\bar{n}_{s} \cdot{ }^{B} \bar{V}^{C}+\left(\overline{G R} \times \bar{n}_{z}\right) \cdot{ }^{B} \bar{\omega}^{p}}{\mu_{i, 2}}
$$

where $\mu_{i, s}$ is the $Z$-component of the vector $\bar{\mu}_{i}$. Also, let

$$
\mu_{i, x}^{\prime}=\frac{\mu_{i, x}}{\mu_{i, \Sigma}}, \quad \mu_{i, y}^{\prime}=\frac{\mu_{i, y}}{\mu_{i, z}}
$$

where $\mu_{i, x}$ and $\mu_{i, v}$ are the $X$ and $Y^{\prime}$-components of vector $\bar{\mu}_{i}$ respectively. Substituting equation (10) into equation (7), and solving for the $\mathrm{X}$-component of the resulting equation, we obtain

$$
\begin{aligned}
{ }^{B} V_{x}^{R_{1}=} & \left(\bar{n}_{x}-\mu_{1, x}^{\prime} \bar{n}_{z}\right) \cdot{ }^{B} \bar{V}^{C}+ \\
& {\left[\left(\overline{G R_{1}} \times \bar{n}_{x}\right)-\mu_{i, x}^{\prime}\left(\overline{G R_{1}} \times \bar{n}_{z}\right)\right] \cdot{ }^{B} \bar{\omega}^{P} }
\end{aligned}
$$

where ${ }^{B} V_{x}^{R_{1}}$ is the $X$-component of vector ${ }^{B} \nabla^{R_{1}}$ and $\tilde{n}_{x}$ is a unit vector in the $X$-direction. Similarly, we can obtain the following equation

$$
\begin{aligned}
{ }^{B} V_{y}^{R_{1}=} & \left(\bar{n}_{y}-\mu_{i, y}^{\prime} \bar{n}_{z}\right) \cdot{ }^{B} \overline{V^{G}}+ \\
& {\left[\left(\overline{G R_{1}} \times \bar{n}_{y}\right)-\mu_{1, y}^{\prime}\left(\overline{G R_{i}} \times \bar{n}_{z}\right)\right] \cdot{ }^{B} \bar{\omega}^{P} }
\end{aligned}
$$

where ${ }^{B} V_{v}^{R_{1}}$ is the $Y^{\prime}$-component of vector ${ }^{B} \bar{V}^{R_{1}}$ and $\bar{n}_{y}$ is a unit vector in the $X^{\prime}$-direction.

Let us define the $6 \times 1$ twist vector of the platform $(\dot{\bar{x}})$ as

$$
\dot{\bar{x}}=\left[\begin{array}{c}
B \bar{V}^{C} \\
{ }^{B} \bar{\omega}^{P}
\end{array}\right]
$$

If the $6 \times 1$ vector of velocity components at the lower ends of the limbs $(\dot{\bar{q}})$ is given by

$$
\dot{\bar{q}}=\left({ }^{B} \bar{V}_{x}^{R_{1}},{ }^{B} \bar{V}_{y}^{R_{1}},{ }^{B} \bar{V}_{x}^{R_{2}},{ }^{B} \bar{V}_{y}^{R_{2}},{ }^{B} \bar{V}_{z}^{R_{3}},{ }^{B} \bar{V}_{y}^{R_{3}}\right\}^{T}
$$

Then, we can define the $6 \times 6$ Jacobian matrix $(\mathrm{J})$ by

$$
\dot{\bar{q}}=\mathrm{J} \dot{\bar{x}}
$$

Referring to equations (12) and (12), we can express the Jaco. bian matrix as

$$
J=\left[\begin{array}{cl}
\left(\bar{n}_{x}-\mu_{1, x}^{\prime} \bar{n}_{z}\right)^{T} & \left(\left(\overline{G R_{1}} \times \bar{n}_{x}\right)-\mu_{1, x}^{\prime}\left(\overline{G R_{1}} \times \bar{n}_{z}\right)\right)^{T} \\
\left(\bar{n}_{y}-\mu_{1, y}^{\prime} \bar{n}_{z}\right)^{T} & \left(\left(\overline{G R_{1}} \times \bar{n}_{y}\right)-\mu_{1, y}^{\prime}\left(\overline{G R_{1}} \times \bar{n}_{z}\right)\right]^{T} \\
\left(\bar{n}_{x}-\mu_{2, x}^{\prime} \bar{n}_{z}\right)^{T} & {\left[\left(\overline{G R_{2}} \times \bar{n}_{z}\right) \cdots \mu_{2, x}^{\prime}\left(\overline{G R_{z}} \times \bar{n}_{z}\right)\right]^{T}} \\
\left(\bar{n}_{y}-\mu_{2, y}^{\prime} \bar{n}_{z}\right)^{T} & \left(\left(\overline{G R_{2}} \times \bar{n}_{y}\right)-\mu_{2, y}^{\prime}\left(\overline{G R_{2}} \times \bar{n}_{z}\right)\right]^{T} \\
\left(\bar{n}_{x}-\mu_{3, x}^{\prime} \bar{n}_{z}\right)^{T} & \left(\left(\overline{G R_{3}} \times \bar{n}_{z}\right)-\mu_{3, x}^{\prime}\left(\overline{G R_{3}} \times \bar{n}_{z}\right)\right]^{T} \\
\left(\bar{n}_{y}-\mu_{3, y}^{\prime} \bar{n}_{y}\right)^{T} & {\left[\left(\overline{G R_{3}} \times \bar{n}_{y}\right)-\mu_{3, y}^{\prime}\left(\overline{G R_{J}} \times \bar{n}_{z}\right)\right]^{T}}
\end{array}\right]
$$

where superscript $T$ denotes transpose.

Note that due to dualities of parallel and serial manipula. tors (Wialdron and Hunt, 1987), we have defined the Jacobian matrix as the transformation which maps the Cartesian veloci. ties to joint rates. This is a common practice among most researchers who have studied parallel manipulators (e.g.; Gosselin and Angeles, 1988, 1989; Gosselin, 1990; A rai et al., 1990).

\subsection{Stiffness Analysis}

From equation (15), we can conclude that

$$
\overline{\delta q}=j \overline{\delta x}
$$

where $\overline{\delta q}$ and $\overline{\delta x}$ represent infinitesimal displacements at the lower ends of the limbs and at the center of the platform, respectively. Equation (15) and the principle of virtual work can be used to derive the following equation (Asada and Slotine, 1986).

$$
\bar{F}=\mathrm{J}^{\mathrm{T}} \bar{f}
$$

where

$$
\bar{F}=\left[\begin{array}{l}
\bar{F}_{P} \\
\bar{M}_{P}
\end{array}\right]
$$

and

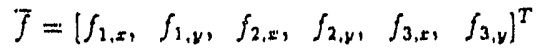

Vectors $\bar{F}_{p}$ and $\bar{M}_{p}$ in equation (19) represent the force and moment applied to the platform. Also, $f_{i, x}$ and $f_{i, y}$ in equation (20) are the $X$ and $Y$-components of the actuator force applied at point $R_{i}$. The aciuator forces and displacements at the lower ends of the limbs can be related by the following equation.

$$
\bar{f}=k \bar{\delta} q
$$

where $k$ is a $6 \times 6$ diagonal matrix whose elements have units of force per urit length. Substituting equation (17) into equation (21) and the resulting equation into equation (18), yields

$$
\overline{\mathcal{F}}=\mathrm{J}^{T} \mathrm{~kJ} \overline{\delta x}
$$

If $k$ represents the stiffness of each bilinear stepper motor (ac. tuator) in the $X$ and $Y$ directions, then the diagonal elements of $k$ are all equal to $k$. Therefore, the stiffness matrix for the platform (K) can be expressed as

$$
\mathrm{K}=k \mathrm{~J}^{T} \mathrm{~J}
$$

\begin{tabular}{|c|c|c|c|c|c|c|}
\hline \multicolumn{7}{|c|}{$\mathrm{K}^{+}=$} \\
\hline \multirow{6}{*}{$3 k$} & 1 & 0 & 0 & 0 & $\frac{(2 p-v) \zeta}{2(v-p)}$ & 0 \\
\hline & 0 & 1 & 0 & $\frac{(v-2 p) s}{2(v-p)}$ & 0 & 0 \\
\hline & 0 & 0 & $\frac{c^{2}}{(v-p)^{2}}$ & 0 & 0 & 0 \\
\hline & 0 & $\frac{(\nu-2 \varepsilon) S}{2(\nu-p)}$ & 0 & $\frac{\left(u^{2}-3 p v+2 p^{2}\right) K^{2}}{2(v-p)^{2}}$ & 0 & 0 \\
\hline & $\frac{(2 p-\nu) s}{2(\nu-p)}$ & 0 & 0 & 0 & $\frac{\left(v^{2}-2 p v+2 p^{3}\right) S^{2}}{2(v-p)^{2}}$ & 0 \\
\hline & 0 & 0 & 0 & 0 & 0 & $\nu^{2}$ \\
\hline
\end{tabular}

Note that $K$ is a symmetric, positive semidefinite matrix. Elements of the lower triangular portion of $K / k(\breve{K})$ are given in Appendix A.

\subsection{Central Stiffress_Matrix}

In this subsection, the stiffness matrix at the central configura. tion of the minirnanipulator workspace (central stiffness matrix) will be derived. The ceniral configuration is defined as the configuration where

1. The platform is not rotated with respect to the base.

2. The centroid of triangle $P_{1} P_{2} P_{3}$ (platform) is directly on top of the centroid of triangle $D_{1} D_{2} D_{3}$, i.e. $X_{G}=Y_{G}=0$.

Let $\left|\overline{G P_{i}}\right|=p$. Also, at the ceniral configuration, let $\left|\overline{O R_{i}}\right|=v$ and $Z_{G}=\zeta$. Using equations (16) and (23), the stiffness matrix at the central configuration $\left(\mathrm{K}^{+}\right)$is found to be 
It is desirable to eliminate the off-diagonal terms which couple the forces (moments) applied along (about) the $X$ and $Y$ axes to the rotations (translations) about (along) the $Y$ and $X$ axes, respectively. Fortunately, this can be casily accomplished by setting

$$
p=\frac{\nu}{2}
$$

In other words, the platform (triangle $P_{1} P_{2} P_{3}$ ) should be onehalf of triangle $R_{1} R_{2} R_{3}$ at the central configuration. The above result is sirnilar to that obtained by Kerr (1989) in designing a Stewart-platform-based force and torque transducer. If the condition expressed in the above equation is satisfied, then

$$
\zeta^{2}=r^{2}-p^{2}
$$

where $r$ is the length of any limb. If equations (25) and (26) are used to substitute for $\nu$ and $\zeta$ in equation (24), matrix $\mathrm{K}^{+}$ redices to

$$
K^{*}=3 k\left[\begin{array}{ccccc}
1 & & & 0 & \\
& 1 & & & \\
& \left(r^{2}-p^{2}\right) / p^{2} & & & \\
& & r^{2}-p^{2} & & \\
& 0 & & r^{2}-p^{2} & \\
& 0 & & & 4 p^{2}
\end{array}\right]
$$

The above equation can be used to determine the relative dimensions of the minimaripulator so that desirable characteristics can be obtained. Note that dimension $\nu$ (the independent variable) can be determined trom other requirements and constraints such as maximizing the workspace and the upper bound on size of the base plate.

For some applications, it may be desirable to maximize one or more of the diagonal elements of $\mathrm{K}^{*}\left(K_{i, 1}^{*}, K_{i, 2}^{*}, \ldots, K_{6,6}^{*}\right)$. For other applications, the designer may be interested in isotropic stifiness properties. Note that it is not possible to make all of the diagonal stiffness terms equal to one another. However, it will be shown that it is possible to obtain isotropic direct stiffness or isotropic torsional stiffness.

To move the platform in the $X$ or $Y$-direction, the lower ends of al! three limbs should also move in the $\mathrm{X}$ or Y-direction. As a result, elements $K_{i, 1}$ and $K_{i, 2}$ are constants. Element $K_{3,3}$ is proportiona! to tangent-squared of the angle between any of the limbs and the base plane. The closer this angle to 90 denrees, the larger the direct stiffness in the Z-direction. Stiffness terms $K_{3,3}^{*}, K_{i, 4}$, and $K_{j, 5}$ are functions of $t$ wo design variables $(r$ and p). However, $K_{6,6}^{\prime}$ is only dependent on variable $p$ (circumradius of the platform). This is related to the fact that in order to rotate the platform about the Z-axis, the lower ends of the limbs should move on a circle, which passes through thern, in the same direction and by an equal amount.

The first three diagonal terms of the $\mathrm{K}^{*}$ matrix are direct, stiffness terms. Equation (27) shows that by setting

$$
r=\sqrt{2} p
$$

we can obtain equal direct stiffness values in the $X, Y$, and $Z$ directions. At this configuration, the angle between any of the limbs and the base plane becornes equal to 45 degrees.

The last three diagonal terms of the $\mathrm{K}^{*}$ matrix are torsional stiffness terms. Referring to equation (27), we notice that by selting

$$
r=\sqrt{5} p
$$

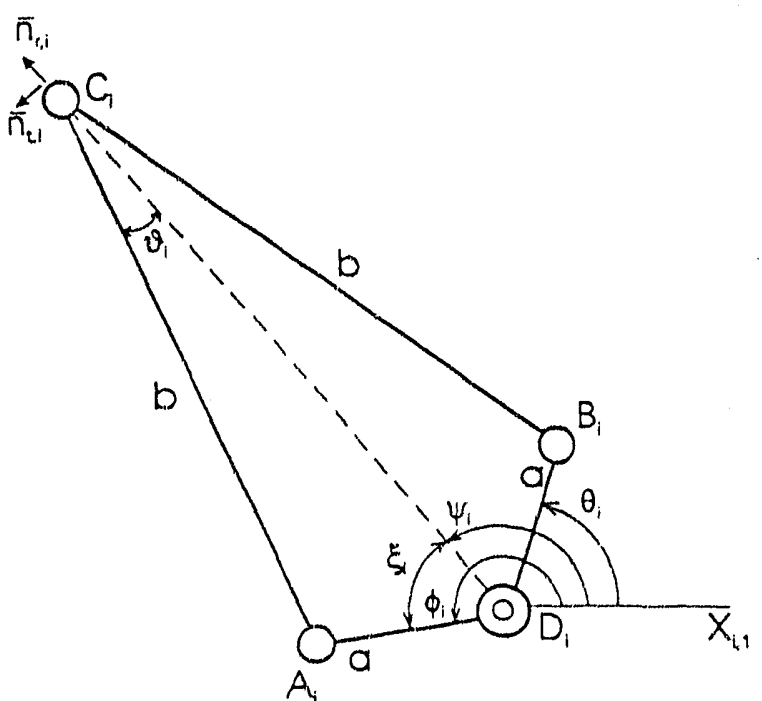

Figure 6: Parameters used in velocity analysis of drivers

we can obtain equal torsional stiffness values in the $X, Y$, and $Z$ directions. At this configuration, the angle between any of the limbs and the base plane becomes equal to 63.43 degrees.

\section{TYPE 2 MINIMANIPULATOR}

In this section, expressions for the Jacobian and stiffness matrices of the Type 2 minimanipulator are derived.

\subsection{Jacobian Analysis}

Figure 6 shows a simplified five-bar driver. Let $\theta_{i}$ and $\phi_{i}$ (driver input angles) be the angles from the positive $X$-axis to the vectors $\overline{D_{i} B_{i}}$ and $\bar{D}_{i} \bar{A}_{i}$, respertively, measured about the positive $Z$-axis. $D_{i} B_{i}$ and $D_{i} A_{i}$ are the inpiat links of the driver and vector $\overline{D_{1} X_{1,1}}$ is parallel to the positive $X$-axis. In the following analysis, we assume that $\phi_{i} \geq 0_{i}$ (if $\phi_{1}<0_{1}, 360$ degrees is added to $\left.\phi_{i}\right)$. In addition. only one branch of a driver is considered, because the other branch can be realized only by disassembling and reassembling the driver. From the driver geometry, we can write

$$
\left|\overline{D_{i} C_{i}}\right|=a \cos \xi_{i}+b \cos v_{i}
$$

where $\xi_{i}$ is the angle of line $D_{i} C_{i}$ with line $D_{i} B_{i}$ or line $D_{i} A_{i}$ and $v_{i}$ is the angle of line $C_{i} D_{i}$ with line $C_{i} B_{i}$ or line $C_{i} A_{i}$. Applying the law of sines to triangle $D_{i} A_{i} C_{i}$, we get

$$
\frac{\sin \vartheta_{i}}{a}=\frac{\sin \xi_{i}}{b}
$$

or

$$
\cos \vartheta_{i}=\sqrt{1-(a / b)^{2} \sin ^{2} \xi_{i}}
$$

From equations (28) and (30), we conclude that

$$
\overline{D_{1} C_{1}}=\left[a \cos \dot{\xi}_{i}+b \sqrt{1-(a / b)^{2} \sin ^{2} \xi_{i}}\right] \ddot{n}_{r, 1}
$$

where $\bar{n}_{r, 4}$ is a unit vector in the direction of vector $\overline{D_{i} C_{1}}$. 
Let $\psi_{i}$ be the angle from the positive $X$-axis to the vector $\overline{D_{i} C_{i}}$ measured about the positive $Z$-axis. Also, as shown in Figure 6 , let the unit vector $\ddot{n}_{t, i}$ be at 90 degrees to the unit vector $\vec{n}_{r, i}$, measured about the positive $Z$-axis. Taking the timederivative of both sides of equation (3i), with respect to the fixed reference frame, we obtain

$$
\left[\begin{array}{l}
{ }^{B} V_{r}^{C_{1}} \\
{ }^{B} V_{t}^{C_{1}}
\end{array}\right]=\Delta^{(i)}\left[\begin{array}{c}
\dot{\xi}_{i} \\
\dot{\psi}_{i}
\end{array}\right]
$$

where $\Delta^{(i)}$ is a $2 \times 2$ diagonal matrix whose diagonal elements are given by

$$
\begin{aligned}
& \Delta_{i, 1}^{(i)}=-b\left[(a / b) \sin \xi_{i}+(a / b)^{2} \frac{\sin \xi_{i} \cos \xi_{i}}{\sqrt{1-(a / b)^{2} \sin ^{2} \xi_{i}}}\right] \\
& \Delta_{2,2}^{(i)}=b\left[(a / b) \cos \xi_{i}+\sqrt{1-(a / b)^{2} \sin ^{2} \xi_{i}}\right]
\end{aligned}
$$

and $\left({ }^{B} V_{r}^{C C_{1}},{ }^{B} V_{t}^{C C_{1}}\right)$ are the radial (in the $\bar{n}_{r, i}$ direction) and tangential (in the $\bar{n}_{t, i}$ direction) components of the velocity of point $C_{i}$. In addition, $\dot{\xi}_{i}$ and $\dot{\psi}_{i}$ denote time-derivatives of angles $\xi_{1}$ and $\psi_{i}$, respectively. Note that $\dot{\xi}_{i}$ and $\dot{\psi}_{i}$ are related to the input speeds $\left(\dot{\theta}_{i}\right.$ and $\left.\dot{\phi}_{i}\right)$ by the following linear relationships.

$$
\begin{aligned}
& \dot{\psi}_{i}=\left(\dot{\phi}_{i}+\dot{0}_{i}\right) / 2 \\
& \dot{\xi}_{i}=\left(\dot{\phi}_{i}-\dot{0}_{i}\right) / 2
\end{aligned}
$$

Equations (32) - (36) show that for a given $b$, the smaller the ratio $a / b$, the higher the speed reduction (mechanical advantage) of the driver in the radial direction.

Substituting equations (35) and (36) into cquation (32) and simplifying, we obtain

$$
\left[\begin{array}{c}
\dot{\theta}_{i} \\
\dot{\phi}_{i}
\end{array}\right]=\left[\begin{array}{cc}
-1 / \Delta_{1,1}^{(1)} & 1 / \Delta_{2,2}^{(i)} \\
1 / \Delta_{1,1}^{(i)} & 1 / \Delta_{2,2}^{(i)}
\end{array}\right]\left[\begin{array}{l}
{ }^{B} V_{r}^{C_{1}} \\
{ }^{{ }^{2}} V_{1}^{C_{1}}
\end{array}\right]
$$

We can also write

$$
\left[\begin{array}{l}
{ }^{B} V_{r}^{C_{1}} \\
{ }^{B} V_{1}^{C_{1}}
\end{array}\right]=\left[\begin{array}{cc}
C \psi_{i} & S \psi_{1} \\
-S \psi_{1} & C \psi_{1}
\end{array}\right]\left[\begin{array}{l}
{ }^{B} V_{x}^{C_{1}} \\
{ }^{B} V_{v}^{C_{1}}
\end{array}\right]
$$

where $\left({ }^{B} V_{x}^{C_{1}},{ }^{B} V_{\nu}^{C_{1}}\right)$ are the components of the velocity of point $C_{1}$ in the $X$ and $Y$ directions, respectively; $C \psi_{i}=\cos \psi_{i}$ and $S \psi_{i}=\sin \psi_{1}$. Equations (37) and (38) imply that

$$
\left[\begin{array}{c}
\dot{\theta}_{i} \\
\dot{\phi}_{i}
\end{array}\right]=\mathrm{J}_{\mathrm{d}}^{(i)}\left[\begin{array}{l}
B \\
{ }^{B} V_{C_{1}}^{C_{1}} \\
B V_{y}^{C_{1}}
\end{array}\right]
$$

where

$$
\mathrm{J}_{\mathrm{d}}^{(i)}=\left[\begin{array}{cc}
-C \psi_{i} / \Delta_{1,1}^{(i)}-S \psi_{i} / \Delta_{2,2}^{(i)} & -S \psi_{i} / \Delta_{1,1}^{(i)}+C \psi_{i} / \Delta_{2,2}^{(i)} \\
C \psi_{i} / \Delta_{1,1}^{(i)}-S \psi_{i} / \Delta_{2,2}^{(i)} & S \psi_{i} / \Delta_{1,1}^{(i)}+C \psi_{i} / \Delta_{2,2}^{(i)}
\end{array}\right]
$$

Let $\dot{\bar{\theta}}$ be the $6 \times 1$ vector of input joint rates, i.e.

$$
\dot{\overline{0}}=\left[\begin{array}{llllll}
\dot{0}_{2}, & \dot{\phi}_{1}, & \dot{0}_{2}, & \dot{\phi}_{2}, & \dot{\theta}_{3}, & \dot{\phi}_{3}
\end{array}\right]^{T}
$$

Since ${ }^{B} V_{z}^{R_{1}}={ }^{B} V_{z}^{C_{1}}$ and ${ }^{B} V_{y}^{A_{1}}={ }^{B} V_{y}^{C_{1}}$, we can write

$$
\dot{\overline{0}}=\mathrm{J}_{\mathrm{a}} \dot{\bar{q}}
$$

where $\dot{q}$ is defined in equation (14) and $J_{d}$ is the $6 \times 6$ Jacobian for the drivers which is given by

$$
J_{d}=\left[\begin{array}{ccc}
J_{d}^{(1)} & & 0 \\
0 & J_{d}^{(2)} & \\
0 & & J_{d}^{(3)}
\end{array}\right]
$$

The overall Jacobian for a Type 2 minimanipulator $(\hat{J})$ is defined by

$$
\dot{\bar{\theta}}=\dot{\jmath} \dot{\bar{x}}
$$

where $\dot{\bar{x}}$ is defined in equation (13). Referring to equations (15) and (42), we can see that

$$
\hat{J}=J_{\mathrm{d}} \mathrm{J}
$$

where $J$, which can be found from equation (16), represents the Jacobian for the main portion (platform and limbs) of the minimanipulator.

\subsection{Stiffness Analysis}

Equation (44) implies that

$$
\overline{\delta \theta}=\hat{\mathbf{j}} \overline{\delta x}
$$

where $\overline{\delta \theta}$ and $\overline{\delta x}$ represent infinitesimal displacements of the actuated joints and the platform, respectively. Let $\bar{\tau}$ be the $6 \times 1$ vector of input joint torques. Similar to equation (18), we can write

$$
\bar{F}=\hat{\mathfrak{J}}^{T} \bar{T}
$$

where $\overline{\mathcal{F}}$ is defined in equation (19). Input actuator torques and displacements are related by

$$
\bar{\tau}=\hat{k} \bar{\delta} \overline{0}
$$

where $\hat{k}$ is a $6 \times 6$ diagonal matrix whose elements represent stiffness of the actuators. Substituting equation (46) into equation (48) and the resulting equation into equation (47), yields

$$
\bar{F}=\hat{\jmath}^{\top} \hat{k} \hat{\jmath} \bar{\delta}
$$

The above equation and equation (45) show that the stiffness matrix for the platform $(\hat{K})$ is given by

$$
\hat{\mathrm{K}}=\hat{\mathrm{J}}^{T} \hat{\mathrm{k}} \hat{\mathrm{J}}=\mathrm{J}^{T} \mathrm{~J}_{\mathrm{d}}{ }^{T} \hat{\mathrm{k}} \mathrm{J}_{\mathrm{d}} \mathrm{J}
$$

\section{DESIGN GUIDELINES}

Based on the results of subsection 3.3, the following design guidelines can be established for the Type 1 minimanipulator.

- The central stiffness matrix can be diagonalized (decoupled) by making the platform (triangle $P_{1} P_{2} P_{3}$ ) one-half the size of the triangle passing through the lower ends of the limbs, i.e. $p=v / 2$.

- If the central stiffness matrix is decoupled, then

- Direct stiffness isotropy can be obtained by making the limb length equal to $\sqrt{2}$ times the circumradius of the platform, i.e. $r=\sqrt{2} p$.

- Torsional stiffness isotropy can be obtained by mak. ing the limb length equal to $\sqrt{5}$ times the circumradius of the platform, i.e. $r=\sqrt{5} p$. 
- The larger the ratio of the limb length to the platform circumradius $(r / p)$, the larger the direct stiffness in the Z-direction.

- For a given platform size, the larger the limb length, the larger the torsional stiffness values in the $X$ and Y-directions.

- For a given limb length, the larger the platform size, the smaller the torsional stiffness values in the $\mathrm{X}$ and $Y$-directions, and the larger the torsional stifiness in the Z-direction.

Note that a minimanipulator will be at or near the center of its workspace during most of its operations. Therefore, establishing design guidelines based on the central stiffness matrix is justified.

The main portion (platform and limbs) of the Type $2 \mathrm{~min}$. imanipulator is the same as that of the Type 1 minimanipulator. Therefore, the above design guidelines can also be applied to the Type 2 minimanipulator. In addition, based on the results of subsection 4.1, the following design guideline can be established for the five-bar drivers of the Type 2 minimanipulator.

- The smaller the ratio of the input link length to the output link length of a driver $(a / b)$, the higher the stiffness of the minimanipulator.

\section{SUMMARY}

In this paper, the Jacobian and stiffness matrices of two types of three-limbed, six-DOF parallel minimanipulators are derived. It is shown that the stiffness matrix at the central configuration of a Type 1 minimanipulator workspace can be decoupled, if the platform size is made half of the size of the triangle passing through the lower ends of the limbs. It is also shown that, at the central configuration of a Type 1 minimanipulator, ratio of the limb length to the platform circumradius must be equal to $\sqrt{2}$ $(\sqrt{5})$ for obtaining direct (torsional) stiffness isotropy. Finally, guidelines for obtaining high stiffness values and for designing drivers of the Type 2 minimanipulator are established.

\section{ACKNOWLEDGMENTS}

This research was supported in part by the U.S. Department of Energy under Grant DEF05-88ER13977 and in part by the NSF Engineering Research Centers program, NSFD CDR 8803012. The first author gratefully acknowledges the support of NASA/Goddard Space Flight Center. Such supports do not constitute endorsements of the views expressed in the paper by the supporting agencies.

\section{REFERENCES}

- Arai, T., Cleary, K., Nakamura, T., Adachi, H., and Homma, K., 1990, "Design, Analysis and Construction of a

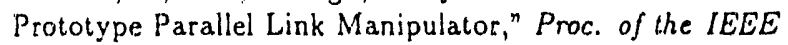
International Workshop on Intelligent Robots and Systems (IROS '90), pp. 205-212.

- Asada, H., and Slotine, J.-J.E., 1986, Robot Analysis and Control. John Wiley and Sons. New York.
- Fichter, E.F., 1986, "A Stewart Platform-based Manipulator: General Theory and Practical Construction," Int. J. of Robotics Research, Vol. 5, pp. 157-182.

- Gosselin, C., and Angeles, J., 1988, "The Optimum Kinematic Design of a Planar Three-Degree-of-Freedom Parallel Manipulator," Trans. ASME, J. of Mech., Transmis., and $A$ uto. in Design, Vol. 110, pp. 35-41.

- Gosselin, C., and Angeles, J., 1989, "The Optimum Kinematic Design of a Spherical Three-Degree-of-Freedom Parallel Manipulator," Trans. ASME, J. of Mech. Transmis., and Auto. in Design, Vol. 111, pp. 202-207.

- Gosselin, C., 1990, "Stiffness Mapping of Parallel Manipulators," IEEE Transactions on Robotics and Automation, Vol. 6, pp. 377-382.

- Griffis, M., and Duffy, J., 1989, "A Forward Displacement Analysis of a Class of Stewart Platforms," J. of Robotic Systems, Vol. 6, pp. 703-720.

- Hudgens, J.C., and Tesar, D., 1988, "A Fully-Parallel Six Degrce-ol-Frcedom Micromanipulator: Kinematic Analysis and Dynamic Model," Trends and Developments in Mechanisms, Machines, and Robotics - Proc. of the 20th Biennial Mechanisms Conference, ASME, New York, DE Vol. 15-3, pp. 29-37.

- Hunt, K.H., 1983, "Structural Kinematics of In-ParallelActuated Robot-Arms," Trans. ASME, J. of Mech., Transmis., and Aulo. in Design, Vol. 105, pp. 705-712.

- Innocenti, C., and Parenti-Castelli, V., 1990, "Direct Position Analysis of the Stewart Platform Mechanism," Mechanism and Machine Theory, Vol. 25, pp. 611-612.

- Kane, T.R., and Levinson, D.A., 1985, Dynamics: Theory and Applications, McGraw-Hill, New York.

- Kerr, D.R., 1989, "Analysis, Properties, and Design of a Stewart-Platform Transducer," Trans. ASME, J. of Mech., Transmis., and Auto. in Design, Vol. 111, pp. 25-28.

- Kohli, D., Lee, S.H., Tsai, K.Y., and Sandor, G.N., 1988, "Manipulator Configurations Based on Rotary-Linear ( $R$ L) Actuators and Their Direct and Inverse Kinematics," Trans. AS.ME, J. of Mech., Transmis., and Auto. in Design, Vol. 110, pp. 397-404.

- Nanua, P., Waldron, K.J., and Murthy, V., 1990, "Direct Kinematic Solution of a Stewart Platform," IEEE Transactions on Robotics and Automation, Vol. 6, pp. 438-444.

- Stewart, D., 1965, "A Platform with Six Degrees of Freedom, ${ }^{n}$ Proc. Institute of Mechanical Engr., London, England, Vol. 180, pp. 371-386.

- Tahmasebi, F., and Tsai, L.W., 1991, "Closed-Form Direct Kinematics Solution of a New Parallel Minimanipulator," Technical Rescarch Report TR 91-92, Systems Research Center, University of Maryiand, College Park. 
- Tsai, L.W., and Tahmasebi, F., 1991 la, "Synthesis and Analysis of a New Class of Six-Degree-of-Freedom Parallel Minimanipulators," Technical Research Report TR 91-83, Systems Research Center, University of Maryland, College Park.

- Tsai, L.W., and Tahmasebi, F., 1991b, "Design and Analysis of a New Six-Degree-of-Freedom Parallel Minimanipulator," Proc. of the 6th International Conference on CAD/CAM, Robotics, and Factories of the Future, Springer-Verlag, Berlin.

- Waldron, K.J., and Hunt, K.II., 1987, "Serics-Parallel Dualities in Actively Coordinated Mechanisms," Proc. of the 4th Int. Symp. on Robotic Research, MIT press, Cam. bridge, MA, pp. 175-181.

- Yeaple, F., 1988, "Choreographed Robots Insert Automotive Parts," Design News, Vol. 44, No. 21, pp. 134-135.

\section{APPENDIX A - LOWER TRIANGULAR ELEMENTS OF $\breve{K}$}

$\check{l}_{1,1}=3$

$\breve{K}_{2,1}=0$

$\ddot{\kappa}_{2,2}=3$

$\check{K}_{3,1}=-\mu_{3, x}^{\prime}-\mu_{2, x}^{\prime}-\mu_{1, x}^{\prime}$

$\check{K}_{3,2}=-\mu_{3, y}^{\prime}-\mu_{2, y}^{\prime}-\mu_{1, y}^{\prime}$

$\check{\mathscr{L}}_{3,3}=\mu_{3, y}^{\prime}{ }^{2}+\mu_{3, x}^{\prime}{ }^{2}+\mu_{2, y}^{\prime}{ }^{2}+\mu_{2, x}^{\prime}{ }^{2}+\mu_{1, y}^{\prime}{ }^{2}+\mu_{1, x}^{\prime}{ }^{2}$

$\check{K}_{4,1}=-\mu_{3, x}^{\prime}\left(Y_{R, 3}-Y_{G}\right)-\mu_{2, x}^{\prime}\left(Y_{R, 2}^{\prime}-Y_{G}\right)-\mu_{1, x}^{\prime}\left(Y_{R, 1}-Y_{G}\right)$

$\check{K}_{4,2}=3 Z_{\mathrm{G}}-\mu_{3, y}^{\prime}\left(Y_{R, 3}-Y_{G}\right)-\mu_{2, Y}^{\prime}\left(Y_{R, 2}-Y_{G}\right)-$ $\mu_{1, Y}^{\prime}\left(Y_{R, 1}-Y_{G}\right)$

$\check{K}_{4,3}=-\mu_{3, y}^{\prime}\left[Z_{G}-\mu_{3, y}^{\prime}\left(Y_{R, 3}-Y_{G}\right)\right]-\mu_{2, y}^{\prime}\left[Z_{G}-\mu_{2, y}^{\prime}\left(Y_{R, 2}-\right.\right.$ $\left.\left.Y_{G}\right)\right]-\mu_{1, Y}^{\prime}\left[Z_{G}-\mu_{1, Y}^{\prime}\left(Y_{R, 1}-Y_{G}\right)\right]+\mu_{3, x}^{\prime}{ }^{2}\left(Y_{R, 3}-Y_{G}\right)+$ $\mu_{2, x}^{\prime}{ }^{2}\left(Y_{R, 2}-Y_{G}\right)+\mu_{1, x}^{\prime}{ }^{2}\left(Y_{R, 1}-Y_{G}\right)$

$\check{K}_{4,4}=\left[Z_{G}-\mu_{3, y}^{\prime}\left(Y_{R, 3}-X_{G}\right)\right]^{2}+\left[Z_{G}-\mu_{2, y}^{\prime}\left(Y_{R, 2}-Y_{G}\right)\right]^{2}+$ $\left[Z_{G}-\mu_{1, y}^{\prime}\left(Y_{R, 1}-Y_{G}\right)\right]^{2}+\mu_{3, x}^{\prime}\left(Y_{R, 3}-Y_{G}\right)^{2}+$ $\mu_{2, x}^{\prime}{ }^{2}\left(Y_{R, 2}-Y_{G}\right)^{2}+\mu_{1, x}^{\prime}{ }^{2}\left(Y_{R, 1}-Y_{G}\right)^{2}$

$\check{K}_{5,1}=-3 Z_{\mathrm{G}}-\mu_{3, x}^{\prime}\left(X_{G}-X_{R, 3}\right)-\mu_{2, x}^{\prime}\left(X_{G}-X_{R, 2}\right)-$ $\mu_{1, x}^{\prime}\left(X_{G}-X_{R, 1}\right)$

$\check{K}_{S, 2}=-\mu_{3, y}^{\prime}\left(X_{G}-X_{R, 3}\right)-\mu_{2, y}^{\prime}\left(X_{G}-X_{R, 2}\right)-\mu_{1, y}^{\prime}\left(X_{G}-X_{R, 1}\right)$

$$
\begin{aligned}
& \check{K}_{S, 3}=-\mu_{3, x}^{\prime}\left[-Z_{G}-\mu_{3, x}^{\prime}\left(X_{G}-X_{R, 3}\right)\right]-\mu_{2, x}^{\prime}\left[-Z_{G}-\mu_{2, x}^{\prime}\left(X_{G}-\right.\right. \\
& \left.\left.\mathrm{X}_{\mathrm{R}, 2}\right)\right]-\mu_{1, x}^{\prime}\left[-Z_{G}-\mu_{1, x}^{\prime}\left(\mathrm{X}_{G}-\mathrm{X}_{\mathrm{R}, 1}\right)\right]+\mu_{3, y}^{\prime}{ }^{2}\left(\mathrm{X}_{G}-\mathrm{X}_{R, 3}\right)+ \\
& \mu_{2, y}^{\prime}{ }^{2}\left(X_{G}-X_{R, 2}\right)+\mu_{1, y}^{\prime}{ }^{2}\left(X_{G}-X_{R, 1}\right) \\
& \check{K}_{5,4}=-\mu_{3, y}^{\prime}\left(X_{G}-X_{R, 3}\right)\left[Z_{G}-\mu_{3, y}^{\prime}\left(Y_{R, 3}-Y_{G}\right)\right]-\mu_{2, y}^{\prime}\left(X_{G}-\right. \\
& \left.X_{R, 3}\right)\left[Z_{G}-\mu_{2, y}^{\prime}\left(Y_{R, 2}-Y_{G}\right)\right]-\mu_{1, y}^{\prime}\left(X_{G}-X_{R, 1}\right)\left[Z_{G}-\right. \\
& \left.\mu_{1, y}^{\prime}\left(Y_{R, 1}-Y_{G}\right)\right]-\mu_{3, x}^{\prime}\left(Y_{R, 3}-Y_{G}\right)\left[-Z_{G}-\mu_{3, x}^{\prime}\left(X_{G}-\right.\right. \\
& \left.\left.X_{R, 3}\right)\right]-\mu_{2, x}^{\prime}\left(Y_{R, 2}-Y_{G}\right)\left[-Z_{G}-\mu_{2, x}^{\prime}\left(X_{G}-X_{R, 2}\right)\right]- \\
& \mu_{1, x}^{\prime}\left(Y_{R, 1}-Y_{G}\right)\left[-Z_{G}-\mu_{1, x}^{\prime}\left(X_{G}-X_{R, 1}\right)\right] \\
& \check{K}_{S, 5}=\left[-Z_{G}-\mu_{3, x}^{\prime}\left(X_{G}-X_{R, 3}\right)\right]^{2}+\left[-Z_{G}-\mu_{2, x}^{\prime}\left(X_{G}-X_{R, 2}\right)\right]^{2}+ \\
& {\left[-Z_{G}-\mu_{1, x}^{\prime}\left(X_{G}-X_{R, 1}\right)\right]^{2}+\mu_{3, y}^{\prime}{ }^{2}\left(X_{G}-X_{R, 3}\right)^{2}+} \\
& \mu_{2, y}^{\prime}{ }^{2}\left(X_{G}-X_{R, 2}\right)^{2}+\mu_{1, y}^{\prime}{ }^{2}\left(X_{G}-X_{R, 1}\right)^{2} \\
& \breve{K}_{6,1}=-Y_{R, 3}-Y_{R, 2}-Y_{R, 1}+3 Y_{G} \\
& \check{K}_{6,2}=X_{R, 3}+X_{R, 2}+X_{R, 1}-3 X_{G} \\
& \check{K}_{6,3}=-\mu_{3, x}^{\prime}\left(Y_{G}-Y_{R, 3}\right)-\mu_{2, x}^{\prime}\left(Y_{G}-Y_{R, 2}\right)-\mu_{1, x}^{\prime}\left(Y_{G}-Y_{R, 1}\right)- \\
& \mu_{3, y}^{\prime}\left(X_{R, 3}-X_{G}\right)-\mu_{2, y}^{\prime}\left(X_{R, Z}-X_{G}\right)-\mu_{1, Y}^{\prime}\left(X_{R, 1}-X_{G}\right) \\
& \check{K}_{6,4}=\left(X_{R, 3}-X_{G}\right)\left[Z_{Q}-\mu_{3, y}^{\prime}\left(Y_{R, 3}-Y_{G}\right)\right]+\left(X_{R, 2}-X_{G}\right)\left[Z_{G}-\right. \\
& \left.\mu_{2, y}^{\prime}\left(Y_{R, 2}-Y_{G}\right)\right]+\left(X_{R, 1}-X_{G}\right)\left[Z_{G}-\mu_{1, y}^{\prime}\left(Y_{R, 1}-Y_{G}\right)\right]- \\
& \mu_{3, x}^{\prime}\left(Y_{G}-Y_{R, 3}\right)\left(Y_{R, 3}-Y_{G}\right)-\mu_{2, x}^{\prime}\left(Y_{G}-Y_{R, 2}\right)\left(Y_{R, 2}-\right. \\
& \left.Y_{G}\right)-\mu_{1, x}^{\prime}\left(Y_{G}-Y_{R, 1}\right)\left(Y_{R, 1}-Y_{G}\right) \\
& \check{h}_{G, 5}=\left(Y_{G}-Y_{R, 3}\right)\left[-Z_{G}-\mu_{3, x}^{\prime}\left(X_{G}-X_{R, 3}\right)\right]+\left(Y_{G}-\right. \\
& \left.Y_{R, 2}\right)\left[-Z_{G}-\mu_{2, X}^{\prime}\left(X_{G}-X_{R, 2}\right)\right]+\left(Y_{G}-Y_{R, 1}\right)\left(-Z_{G}-\right. \\
& \left.\mu_{1, x}^{\prime}\left(X_{G}-X_{R, 1}\right)\right]-\mu_{3, Y}^{\prime}\left(X_{G}-X_{R, 3}\right)\left(X_{R, 3}-X_{G}\right)- \\
& \mu_{2, y}^{\prime}\left(X_{G}-X_{R, 2}\right)\left(X_{R, 2}-X_{G}\right)- \\
& \mu_{1, y}^{\prime}\left(X_{G}-X_{R, 1}\right)\left(X_{R, 1}-X_{G}\right) \\
& \check{I}_{6,6}=\left(Y_{G}-Y_{R, 3}\right)^{2}+\left(Y_{G}-Y_{R, 2}\right)^{2}+\left(Y_{G}-Y_{R, 1}\right)^{2}+ \\
& \left(X_{R, 3}-X_{G}\right)^{2}+\left(X_{R, 2}-X_{G}\right)^{2}+\left(X_{R, 1}-X_{G}\right)^{2}
\end{aligned}
$$

where $\left(X_{R, i}, Y_{R, i}\right)$ are the $(\mathrm{X}, \mathrm{Y})$ coordinates of point $R_{i}$ and $\left(X_{G}, Y_{G}, Z_{G}\right)$ are the coordinates of point $G$. 

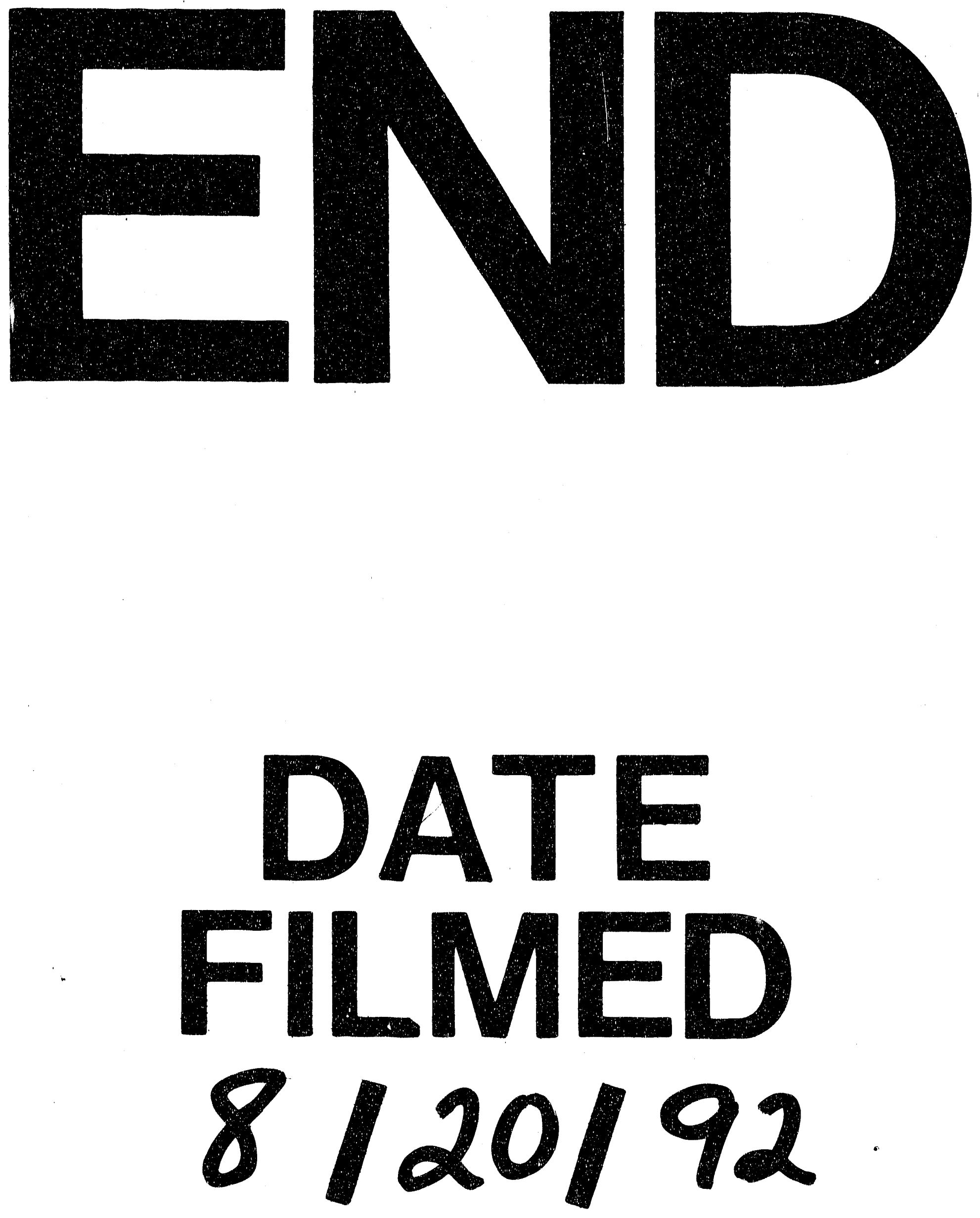
